\title{
Chromosomal breakpoint mapping by arrayCGH using flow-sorted chromosomes
}

\author{
Imke M. Veltman ${ }^{1}$, Joris A. Veltman ${ }^{1}$, Ger Arkesteijn ${ }^{2}$, Irene M. Janssen ${ }^{1}$, \\ Lisenka E. Vissers ${ }^{1}$, Pieter J. de Jong ${ }^{3}$, Ad Geurts van Kessel ${ }^{1}$, and \\ Eric F.P.M. Schoenmakers ${ }^{1}$ \\ ${ }^{1}$ University Medical Center Nijmegen, Nijmegen, The Netherlands, ${ }^{2}$ Veterinary Medi- \\ cine Utrecht University, Utrecht, The Netherlands, and ${ }^{3}$ Children's Hospital Oakland \\ Research Institute, Oakland, CA, USA
}

BioTechniques 355:1066-1070 (November 2003)

Despite the recent completion of the human genome project, the mapping of disease-related chromosomal translocation breakpoints and genes has remained laborious. Here, we describe a novel and rapid procedure to map such translocation breakpoints using flow-sorted chromosomes in combination with array-based comparative genomic hybridization (arrayCGH). To test the feasibility of this approach, we used a t(12;15)(q13;q25)-positive cell line with known breakpoint positions as a model. The derivative 12 chromosomes were flowsorted, labeled, and hybridized to a genome-wide array containing 3648 well-characterized human genomic clones. The exact locations of the breakpoints on both chromosome 12 and 15 could be determined in a single hybridization experiment. In addition, we have tested the minimal amount of material necessary to perform these experiments and show that it is possible to obtain highly reliable profiles using as little as 10,000 flow-sorted chromosomes.

\section{INTRODUCTION}

There is ample evidence that recurrent chromosomal abnormalities, including translocations, are causally related to abnormal development and tumorigenesis. Indeed, the molecular characterization of translocation breakpoints has instrumented in the identification of disease-causing genes and the subsequent elucidation of the underlying molecular defects. In cancer, for example, more than 100 recurring chromosomal translocations are known to trigger the activation of various oncogenes (http://www.ehgonline.net/ translocation.pdf).

Despite the fact that novel technologies have recently expedited the laborious process of positional cloning, such an approach still requires a considerable investment in time and reagents. Current methods may include chromosomal flow-sorting, (reverse) painting, spectral karyotyping, and/or fluorescent in situ hybridization (FISH) (1-4). These technologies, however, lack the resolution required for a rapid identification of breakpoint-spanning genomic sequences and/or genes. In order to overcome these limitations and take advantage of recent technological developments, we developed an alternative method.

Here we show that rapid and reliable high-resolution breakpoint mapping is feasible, using a combination of chromosome flow-sorting and array-based comparative genomic hybridization (arrayCGH) and employing a genomewide array containing 3648 genomic clones. As a proof of principle, we used a $\mathrm{t}(12 ; 15)(\mathrm{q} 13 ; \mathrm{q} 25)$-positive cell line with known breakpoint positions (Reference 5 and unpublished data).

\section{MATERIALS AND METHODS}

\section{Cell Culture and Preparation}

A $\mathrm{t}(12 ; 15)(\mathrm{q} 13 ; \mathrm{q} 25)$-containing cell line was established by Epstein-Barr virus transformation of peripheral blood lymphocytes and cultured in RPMI 1640 medium supplemented with $10 \%$ fetal calf serum, $2 \mathrm{mM}$ L-glutamine, and antibiotics at $37^{\circ} \mathrm{C}$ in a humidified atmosphere containing $5 \% \mathrm{CO}_{2}$. Cells in $\log$ phase were treated for $16 \mathrm{~h}$ with colcemid $(0.05 \mathrm{mg} / \mathrm{mL}$ final concentration) to arrest cells in metaphase. Metaphase chromosomes were subsequently isolated for flow sorting as described previously (1).

\section{Flow Karyotyping and Sorting}

Chromosomes were stained with chromomycin-A3 (CA3) and Hoechst 33258 (Ho) and analyzed on a duallaser beam flow cytometer (FACSVantage $^{\mathrm{TM}}$ SE; Becton Dickinson, Franklin Lakes, NJ, USA). CA3 was excited with an argon ion laser tuned at $458 \mathrm{~nm}$ at $100 \mathrm{~mW}$, and CA3 fluorescence was measured through a 550-nm longpass filter. Ho was excited with an argon ion laser tuned into the UV range (351 and $364 \mathrm{~nm}$ ) at $125 \mathrm{~mW}$ laser power, and Ho fluorescence was measured through two KV 408 filters. The system was triggered on the CA3 fluorescence signal. In total, 300,000 chromosomes were sorted from each cluster (Figure 1) and subsequently purified using the QIAamp ${ }^{\circledR}$ purification kit (Qiagen, Hilden, Germany). DNA obtained in this way was used for arrayCGH analyses. Due to the fact that these small amounts of DNA cannot be measured accurately, we have based the number of chromosome copies on the number that is indicated by the flow cytometer.

\section{Array-Based Comparative Genomic Hybridization}

ArrayCGH was performed essentially as described before $(6,7)$ with minor modifications, including the production of a genome-wide bacterial artificial chromosome (BAC) array, the use of an automated hybridization station, and advanced normalization procedures (8). In brief, the array used in this study contained 3648 colony-purified, FISHverified, phage-tested BAC clones spotted in triplicate, most of which have been fully integrated in the human genome browser (http://genome.ucsc.edu/). Approximately 3000 of these clones cover the genome at a $1 \mathrm{Mb}$ resolution. Additional clones included those mapping to known microdeletion hot-spots and to subtelomeric regions, as well as clones covering certain chromosome regions with a higher resolution than $1 \mathrm{Mb}$, including clones that were generated in the context of a previous positional cloning project performed on the cell line discussed in this paper. The arrayCGH profile of the flow-sorted chromosomes was established through hybridization of different amounts 
of Cy ${ }^{\mathrm{TM}} 3$ dUTP-labeled flow-sorted chromosomes combined with $500 \mathrm{ng}$ Cy5 dUTP-labeled control genomic DNA. After scanning, fluorescence test over reference ratios (T/R) were determined for each clone and subsequently $\log 2$-transformed. These $\log 2$ ratios were centered to 0 by dividing the ratio for each clone by the mean of all ratios except for those mapping to the chromosomes 12 and 15 . The latter approach was taken because there was hardly any signal in the $\mathrm{Cy} 3$ channel for the clones not present on the derivative chromosome 12 [der(12)].

\section{RESULTS AND DISCUSSION}

A $t(12 ; 15)(q 13 ; q 25)-$ positive cell line with previously characterized breakpoint positions (Reference 5 and unpublished data) was used for FACSmediated flow-sorting of the $\operatorname{der}(12)$ and the normal chromosome 12 cluster (Figure 1). Due to the fact that the normal chromosome 12 overlaps with chromosomes 9, 10, and 11 in the flow karyogram, these chromosomes were sorted together.

In an initial test, we used 150,000 copies of the chromosome 9-12 cluster (test) labeled with Cy3 dUTP in combination with $500 \mathrm{ng}$ normal male genomic DNA (reference) labeled with Cy5 dUTP and hybridized the mixture to the genome-wide BAC array. This pilot showed a marked hybridization of the clones mapping to the chromosomes 9-12 in the Cy3 channel, whereas virtually no signals were measured for the clones mapping to the other chromosomes. Due to the fact that the normal Cy5-labeled genomic reference DNA hybridized equally to all clones, the resulting $\log 2 \mathrm{~T} / \mathrm{R}$ values for the clones mapping to the chromosomes 9-12 varied between 2 and 3, whereas the clones mapping outside these chromosomes were centered around 0 (results not shown). This initial test showed that this procedure allows a quick identification of the precise chromosomal regions selected by flow-sorting onto the genome-

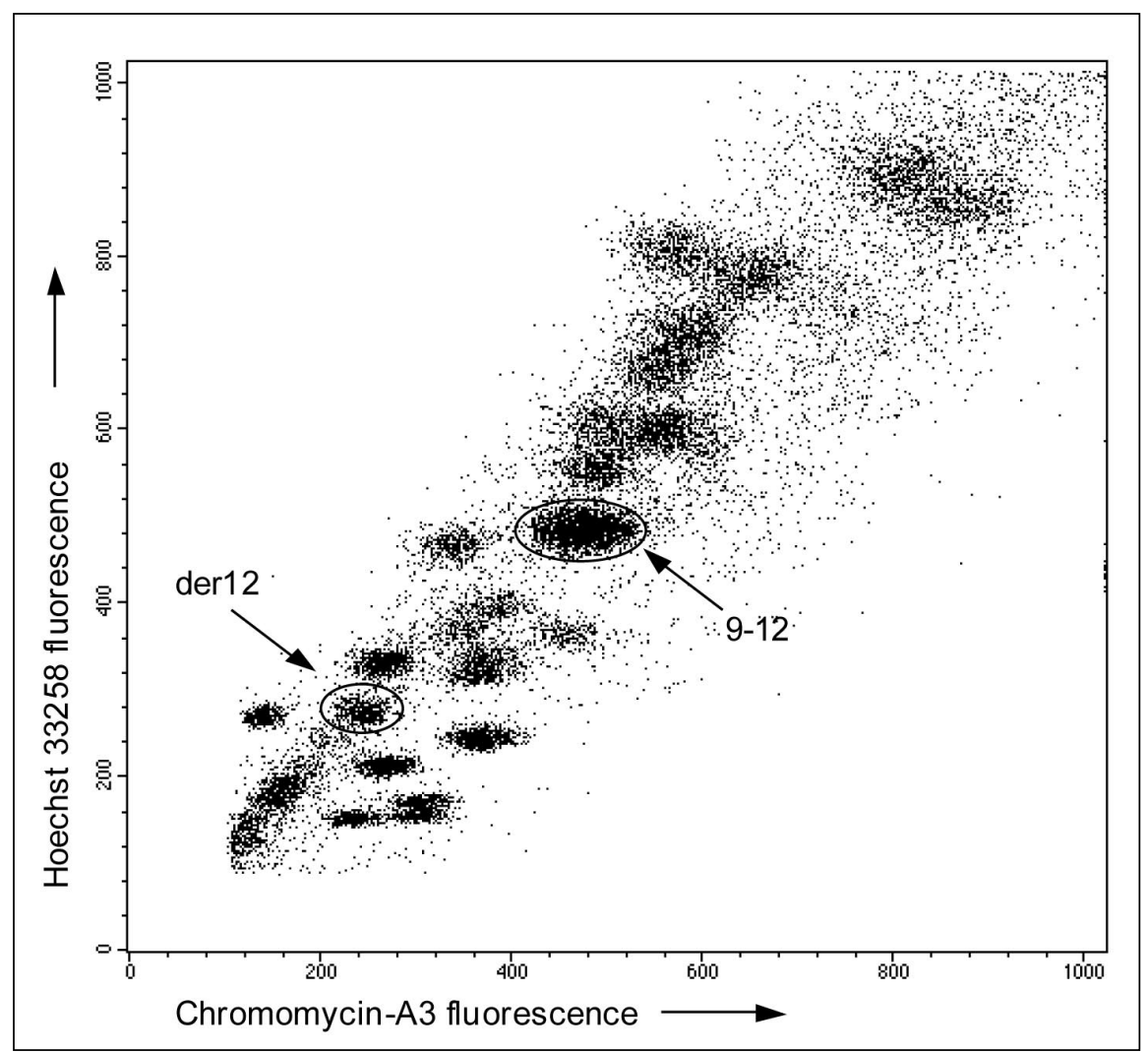

Figure 1. Flow-karyogram of the $\mathbf{t}(\mathbf{1 2} ; \mathbf{1 5})(\mathbf{q 1 3} ; \mathbf{q 2 5})$-positive cell line. The circles mark the selected areas that were flow-sorted: the derivative 12 chromosome [der(12)] and the chromosome 12 cluster, including chromosomes 9, 10, and 11. 


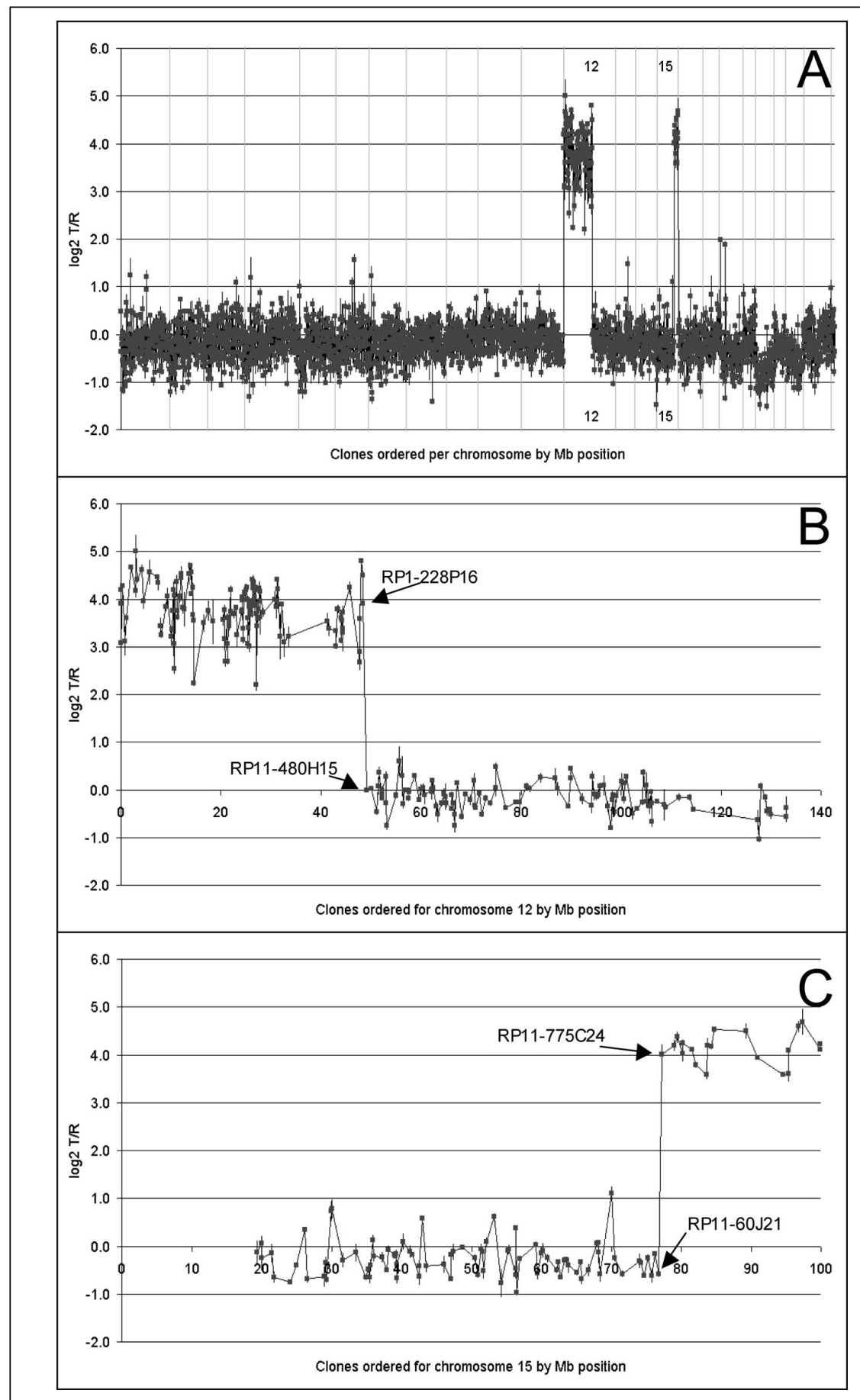

Figure 2. Mapping of the $t(12 ; 15)(q 13 ; q 25)$ by array-based comparative genomic hybridization (arrayCGH) analysis. Profiles are shown after hybridization of 100,000 copies of the derivative 12 chromosome [der(12)] (test), Cy3 dUTP-labeled, versus 500 ng of normal male genomic DNA (reference), Cy5 dUTP-labeled. Dark squares represent mean normalized test over reference (T/R) values for each clone in a $\log 2$ scale, and the vertical lines represent the standard deviations of the replicates per clone. (A) The T/R values of the genomic clones, ordered from chromosome 1 to $\mathrm{Y}$, and within each chromosome by $\mathrm{Mb}$ position as presented in the June 2002 human genome assembly freeze. The vertical lines separate the clones per chromosome. A clear distinction can be made between the clones that hybridize to der(12) (with high T/R values) and the clones that do not (low T/R values). (B) A zoom-in showing the $T / R$ values of the chromosome 12 clones, ordered by Mb position. Indicated are the clones surrounding the breakpoint. (C) T/R values of the chromosome 15 clones, ordered by Mb position. Absence of clones from 0 to approximately $20 \mathrm{Mb}$ is due to the difficulty of mapping, cloning, and sequencing of this region. Indicated are the clones surrounding the breakpoint. wide BAC array. Furthermore, this test also indicates that less flow-sorted chromosomes might be used in these array experiments. Accordingly, hybridizations were performed with 100,000 copies of der(12) labeled with $\mathrm{Cy} 3$ dUTP (test) against $500 \mathrm{ng}$ of normal male genomic DNA labeled with Cy5 dUTP (reference) (Figure 2). The results showed a clear difference in $\mathrm{T} / \mathrm{R}$ values between the clones that map to the der(12) and the clones that do not. The clones from 12pter through clone RP1228 p16 on $12 \mathrm{q} 13.1(48.4 \mathrm{Mb})$ revealed a mean $\log 2 \mathrm{~T} / \mathrm{R}$ value of 3.8 , whereas the clones from RP11-480h15 on 12q13.1 $(49.0 \mathrm{Mb})$ until 12qter displayed a $\log 2$ $\mathrm{T} / \mathrm{R}$ value in the normal range (mean 0 ) (Figure 2B). This result indicates that the breakpoint is positioned in the region encompassing RP1-228p16 and RP11$480 \mathrm{~h} 15$ and correlates perfectly with our recent positional cloning efforts, which revealed that clone RP1-228p16 contains the breakpoint-spanning gene (unpublished data). For chromosome 15 , a similar pattern was observed, with a normal $\log 2 \mathrm{~T} / \mathrm{R}$ value for all clones from 15q12 through clone RP11-60j21 on $15 \mathrm{q} 25(76.8 \mathrm{Mb})$ and a mean $\log 2 \mathrm{~T} /$ $\mathrm{R}$ value of 4.1 for all clones from RP11$775 \mathrm{c} 24$ on $15 \mathrm{q} 25(77.2 \mathrm{Mb})$ until 15 qter (Figure 2C). Again this result perfectly matches our recent data indicating that RP11-775c24 contains the breakpointspanning gene on chromosome 15 .

In order to define the minimal amount of chromosome copies necessary to acquire a reliable arrayCGH profile, 50,000 der(12), 10,000 der(12), and $1000 \operatorname{der}(12)$ copies were hybridized as test DNA together with $500 \mathrm{ng}$ of reference DNA to our genome-wide chip assay. The results showed that hybridizations with 50,000 and 10,000 derivative chromosomes gave profiles similar to Figure 2, whereas for 1000 copies, a clear-cut distinction between der(12) clones and non-der(12) clones was no longer possible. Therefore, the minimal amount of chromosome copies for a reliable arrayCGH profile lies in between 1000 and 10,000 copies. The amount of 10,000 copies equals the chromosome copy number of autosomal chromosomes that are present in approximately $30 \mathrm{ng}$ of genomic DNA from normal diploid human cells, assuming that a normal diploid hu- 
man cell contains about 6 pg of DNA. Interestingly, in a typical arrayCGH experiment, $500 \mathrm{ng}$ of genomic DNA is routinely used. As an alternative for the use of even smaller numbers of flowsorted chromosomes, an amplification step may be included [e.g., degenerate oligonucleotide-primed (DOP) PCR] $(9,10)$. In a preliminary study, we found that this approach is feasible using 1000 copies as input in the PCR, although this needs further optimization (data not shown). Such amplifications may be required for other applications of this method (e.g., delineation of the genomic content of unknown marker chromosomes in primary clinical samples).

This study provides a further example of the power of the arrayCGH technology, which is rapidly becoming the method of choice for high-resolution screening of genomic copy number changes $(7,11-13)$. Arrays with a genome-wide coverage at the $1-3 \mathrm{Mb}$ resolution are becoming more readily available for these kind of applications $(8,9,14)$.

A novel method for rapid mapping of chromosomal translocation breakpoints based on a combination of chromosome flow-sorting and arrayCGH was tested. As a proof of principle, der(12) chromosomes from a known $\mathrm{t}(12 ; 15)(\mathrm{q} 13 ; \mathrm{q} 25)$-positive cell line were flow-sorted and hybridized to a genome-wide chip containing 3648 well-characterized genomic clones. In a single hybridization experiment, using as little as 10,000 copies of the der(12) chromosome, the breakpoints at both chromosome 12 and 15 were fine-mapped, fully in line with our recent positional cloning studies (unpublished data). For chromosome 12, the breakpoint was positioned in the interval RP1-228p16 to RP11-480h15 (an approximate 600-kb interval), and for chromosome 15, the breakpoint was positioned in the interval RP11-60j21 to RP11-775-c24 (an approximate 400$\mathrm{kb}$ interval). This method will greatly facilitate the tedious procedure of positional cloning from balanced chromosomal anomalies. Although we show the applicability of this approach using a cytogenetically characterized translocation, this approach can be extended to all undefined complex chromosomal anomalies. The only prerequisite for using this approach is the necessity to be able to flow-sort aberrant chromosomes separately from their (supposedly) reciprocal translocation products. Currently, we are in the process of preparing a full-coverage $(32 \mathrm{~K})$ genomewide chip (http://bacpac.chori.org/ pHumanMinSet.htm). Once available, we will be able to map translocation breakpoints to a single BAC clone in a single experiment, leading to direct gene identification.

\section{ACKNOWLEDGMENTS}

We thank Kazutoyo Osoegawa and Chik On Choy (BACPAC Resources) for co-establishing the $3.7 \mathrm{~K}$ BAC set and scientific interactions, Walter van der Vliet for array preparations, and Huub Straatman for statistical analyses. Furthermore, we thank Lutgarde Govaerts (Erasmus University, Rotterdam) for supplying us with the $t(12 ; 15)(q 13 ; q 25)$-positive cell line.

\section{REFERENCES}

1.Arkesteijn, G., E. Jumelet, A. Hagenbeek, E. Smit, R. Slater, and A. Martens. 1999. Reverse chromosome painting for the identification of marker chromosomes and complex translocations in leukemia. Cytometry 35: 117-124.

2.Griffin, D.K., D. Sanoudou, E. Adamski, C. McGiffert, P. O'Brien, J. Wienberg, and M.A. Ferguson-Smith. 1998. Chromosome specific comparative genome hybridisation for determining the origin of intrachromosomal duplications. J. Med. Genet. 35:37-41.

3.Heng, H., C. Ye, F. Yang, S. Ebrahim, G. Liu, S. Bremer, C. Thomas, J. Ye, et al. 2003. Analysis of marker or complex chromosomal rearrangements present in pre- and post-natal karyotypes utilizing a combination of G-banding, spectral karyotyping and fluorescence in situ hybridization. Clin. Genet. 63:358-367.

4.Suijkerbuijk, R.F., D. Matthopoulos, L. Kearney, S. Monard, S. Dhut, F.E. Cotter, J. Herbergs, A. Geurts van Kessel, and B.D. Young. 1992. Fluorescent in situ identification of human marker chromosomes using flow sorting and Alu element-mediated PCR. Genomics 13:355-362.

5.Veltman, I., M. van Asseldonk, M. Schepens, H. Stoop, L. Looijenga, C. Wouters, L. Govaerts, R. Suijkerbuijk, and A. Geurts van Kessel. 2002. A novel case of infantile sacral teratoma and a constitutional $\mathrm{t}(12 ; 15)(\mathrm{q} 13 ; \mathrm{q} 25)$ pat. Cancer Genet. Cytogenet. 136:17-22.

6.Veltman, J.A., Y. Jonkers, I. Nuijten, I. Janssen, D. Van, V, E. Huys, J. Vermeesch,
G. Van Buggenhout, et al. 2003. Definition of a Critical Region on Chromosome 18 for Congenital Aural Atresia by ArrayCGH. Am. J. Hum. Genet. 72:1578-1584.

7.Veltman, J.A., E.F. Schoenmakers, B.H. Eussen, I. Janssen, G. Merkx, B. van Cleef, C.M. van Ravenswaaij, H.G. Brunner, et al. 2002. High-throughput analysis of subtelomeric chromosome rearrangements by use of array-based comparative genomic hybridization. Am. J. Hum. Genet. 70:1269-1276.

8.Vissers, L.E., L.B.A. de Vries, I. Janssen, T. Feuth, H. Straatman, W. van der Vliet, E. Huys, P. de Jong, et al. High resolution whole genome microdeletion/duplication screening by arrayCGH. Am. J. Hum. Genet. (In press).

9.Fiegler, H., P. Carr, E.J. Douglas, D.C. Burford, S. Hunt, J. Smith, D.Vetrie, P. Gorman, et al. 2003. DNA microarrays for comparative genomic hybridization based on DOP-PCR amplification of BAC and PAC clones. Genes Chromosomes Cancer 36: 361-374.

10.Telenius, H., A.H. Pelmear, A. Tunnacliffe, N.P. Carter, A. Behmel, M.A. FergusonSmith, M. Nordenskjold, R. Pfragner, and B.A. Ponder. 1992. Cytogenetic analysis by chromosome painting using DOP-PCR amplified flow-sorted chromosomes. Genes Chromosomes Cancer 4:257-263.

11.Pinkel, D., R. Segraves, D. Sudar, S. Clark, I. Poole, D. Kowbel, C. Collins, W.L. Kuo, et al. 1998. High resolution analysis of DNA copy number variation using comparative genomic hybridization to microarrays. Nat. Genet. 20:207-211

12.Pollack, J.R. and V.R. Iyer. 2002. Characterizing the physical genome. Nat. Genet. 32(Suppl.):515-521.

13.Solinas-Toldo, S., S. Lampel, S. Stilgenbauer, J. Nickolenko, A. Benner, H. Dohner, T. Cremer, and P. Lichter. 1997. Matrix-based comparative genomic hybridization: biochips to screen for genomic imbalances. Genes Chromosomes Cancer 20:399-407.

14.Snijders, A.M., N. Nowak, R. Segraves, S. Blackwood, N. Brown, J. Conroy, G. Hamilton, A.K. Hindle, et al. 2001. Assembly of microarrays for genome-wide measurement of DNA copy number. Nat. Genet. 29:263-264.

Received 4 June 2003; accepted 5 August 2003.

Address correspondence to Imke Veltman, Department of Human Genetics, 417, University Medical Center Nijmegen, P.O. Box 9101, 6500 HB Nijmegen, The, Netherlands.e-mail: i.veltman@antrg.umcn.nl 